\title{
Off-resonance intermittent contact mode multi-harmonic scanning force microscopy
}

\author{
M. Penedo ${ }^{1, a)}$ and H. J. Hug ${ }^{1,2}$ \\ ${ }^{1}$ Empa, Swiss Federal Laboratories for Materials Science and Technology, CH-8600 Dübendorf, Switzerland \\ ${ }^{2}$ Department of Physics, University of Basel, CH-4056 Basel, Switzerland
}

(Received 22 February 2018; accepted 28 June 2018; published online 12 July 2018)

\begin{abstract}
A robust off-resonance intermittent contact mode scanning force microscopy technique suitable for operation under different environmental conditions is presented. The technique relies on a multichannel lock-in amplifier to measure multiple high harmonic magnitudes and phases. For distance control, the fundamental harmonic magnitude is used. With this, high intermittent contact frequencies become feasible even with older atomic force microscope data acquisition systems with limited measurement bandwidths, provided high frequency tip-sample distance actuation techniques are used. Suitable higher harmonic magnitude images provide a qualitative materials' contrast. If a sufficiently high number of high harmonic magnitudes and phases are recorded, force-distance curves at all imaged points can be reconstructed. From fitting models of the contact mechanics to force versus tip-sample penetration data, the elastic modulus of the sample can be obtained. Published by AIP Publishing. https://doi.org/10.1063/1.5026657
\end{abstract}

The atomic force microscope (AFM) is a versatile tool for measuring the topography and local mechanical properties of materials. A robust method to assess the latter is the Peak Force operation mode. ${ }^{1,2}$ It allows a precise control of the maximum force the tip applies to the sample, and to map the penetration of the tip into the sample as a function of the applied force. An alternative method is Intermodulation AFM, ${ }^{3,4}$ where the cantilever oscillation is driven at two frequencies close to the cantilever resonance frequency. The non-linear tip-sample interaction then generates high-order frequency products of the two drive frequencies (intermodulation) from which the tip-sample interaction can be calculated.

From the cantilever deflection versus distance data (either measured directly ${ }^{1}$ or reconstructed from the intermodulation data $\left.{ }^{3,4}\right)$, the penetration of the tip into the sample as a function of the force applied by the tip to the sample can be extracted. The elastic modulus of the sample can be obtained from fitting the force-penetration data with appropriate models describing the elastic tip-sample interaction, ${ }^{5}$ provided the geometry of the tip is known. On samples with spatial variations of the elastic modulus, the spatial map of the tip's penetration into the sample can be summed to the output of the z-feedback (as-measured topography) to obtain an improved measure of the sample topography (true topography). Further information on the local sample properties can be deduced from the maximum adhesion force.

In the Peak Force mode, the tip-sample position is sinusoidally modulated with an oscillation amplitude of 50-200 nm, and at frequencies of typically $1-2 \mathrm{kHz}$. The average sample position is adjusted such that a repeated contact of the tip with the sample occurs [Fig. 1(a)], and multiple cantilever deflection versus tip-sample travel curves are recorded [Fig. 1(b)]. The corresponding time trace is analyzed in real-time to determine the maximum force which is then kept constant by the $\mathrm{z}$ -

\footnotetext{
a) Author to whom correspondence should be addressed: marcos.penedo-garcia@ empa.ch
}

feedback, the penetration into the sample as a function of the applied force, and the maximum adhesion force. Such a precise control of the tip-sample force is particularly useful when imaging soft samples with locally varying elastic moduli, and specifically for imaging biological samples. ${ }^{10}$ However, particularly for the latter, higher scan-speeds are beneficial. Nievergelt et al. ${ }^{10}$ have demonstrated that higher oscillation frequencies and consequently higher scan-speeds can be obtained with their ingenious dual actuator design. Modulation frequencies of up to $31.5 \mathrm{kHz}$ were used, but because of the $500 \mathrm{kHz}$ sampling rate of their acquisition card, only 16 points per force-distance curve were recorded. Here, several disadvantages of the peak force method become apparent. First, 16 point per force curves will not permit a low noise detection of the peak force, limiting the use of this signal for the operation of the z-feedback. Second, the $31.5 \mathrm{kHz}$ tapping frequency limits the pixel frequency to 31500 pixels per second even if only
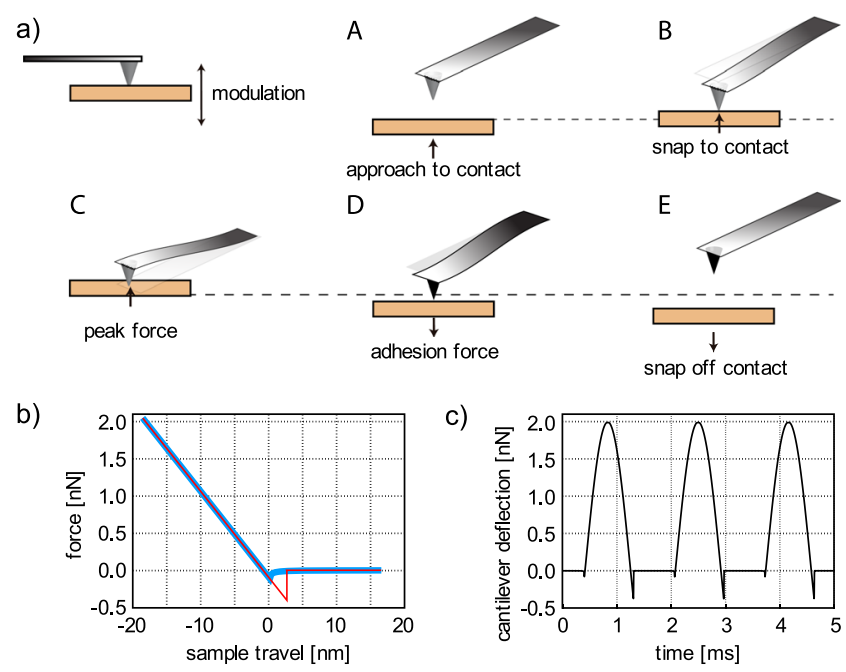

c)

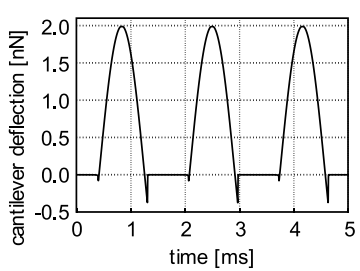

FIG. 1. (a) Principle of an off-resonance intermittent contact mode (also used for the Peak Force mode). (b) Schematical cantilever force-distance curve. (c) Schematical time-trace of the cantilever deflection signal. 
1 tap per pixel is used. Operation modes that avoid the acquisition of data points that do not contain useful information on the tip-sample mechanics and provide a low-noise signal for the z-feedback are thus required.

One possible implementation is the intermodulation method, ${ }^{3,4}$ that uses a multi-channel lock-in amplifier (LIA) ${ }^{14}$ to simultaneously record a sufficiently high number of intermodulation magnitudes and phases. From these data, it is possible to reconstruct the force-distance curves, ${ }^{6}$ but sophisticated mathematical models are required. Further, the intermodulation magnitudes decay rapidly with increased harmonic number. Hence, only magnitudes and phases of intermodulation frequencies within the width of the cantilever resonance frequency peak can be recorded. In vacuum, the latter however can become extremely narrow. This increases the instruments' force (gradient) sensitivity but limits the applicability of the intermodulation method, because the independent detection of the closely-spaced intermodulation frequencies requires small measurement bandwidths of the LIA. This limits the use of the intermodulation method to ambient environments. It is further possible to measure mechanical sample properties with other resonance-based AFM operation modes. ${ }^{7-9}$ However, the high quality factor of the cantilever resonances obtained in vacuum makes the control of the oscillation amplitudes and phases challenging if the tip contacts the sample surface.

Here, we present an alternative method that maps only relevant data (similar as the intermodulation method), but can still assess all local sample properties typically extracted from Peak Force data. We show that the operation mode is robust, i.e., that it works under ambient conditions and in vacuum; that samples with micrometer deep trenches and vertical walls can be imaged; and that atomic-sized steps can be measured. Further, we demonstrate that materials' contrast can be obtained on samples containing metal, silicon, and poly(methyl methacrylate) (PMMA), or polystyrene (PS) and polyethylene polymers.

In the off-resonance intermittent contact mode presented here, the tip-sample position is sinusoidally modulated with an amplitude and frequency similar to that used in the Peak Force mode. The deflection signal resulting from the intermittent contact [Fig. 1(c)] is then sent to a multi-channel lock-in amplifier (HF2LI from Zurich Instruments ${ }^{11}$ ) tuned to the first and/or higher harmonics of the modulation signal. The magnitude of the first (or a suitable higher) harmonic magnitude can then used as a proxy for the average tip-sample z-position, i.e., the latter is adjusted by the z-feedback such that the chosen harmonic signal remains constant during sample imaging. From simulations, we find that the peak force depends monotonously on the 1st harmonic magnitude signal and is thus an excellent proxy for the tip-sample interaction (see Fig. S2 of the supplementary material).

Figures 2(a)-2(f) show the topographies and crosssections of various samples measured under ambient conditions [Figs. 2(a) and 2(b) and 2(e) and 2(f)] and in a vacuum of about $10^{-6}$ mbar [Figs. 2(c) and 2(d)]. Figures 2(a) and 2(b) shows results obtained on a micro-fabricated sample consisting of materials with a very different elastic modulus [naturally oxidized silicon $\left(\mathrm{SiO}_{2}\right)$, poly(methyl methacrylate) (PMMA), and gold], and a topography dominated by deep micro-fabricated trenches of several $100 \mathrm{~nm}$ depth and vertical
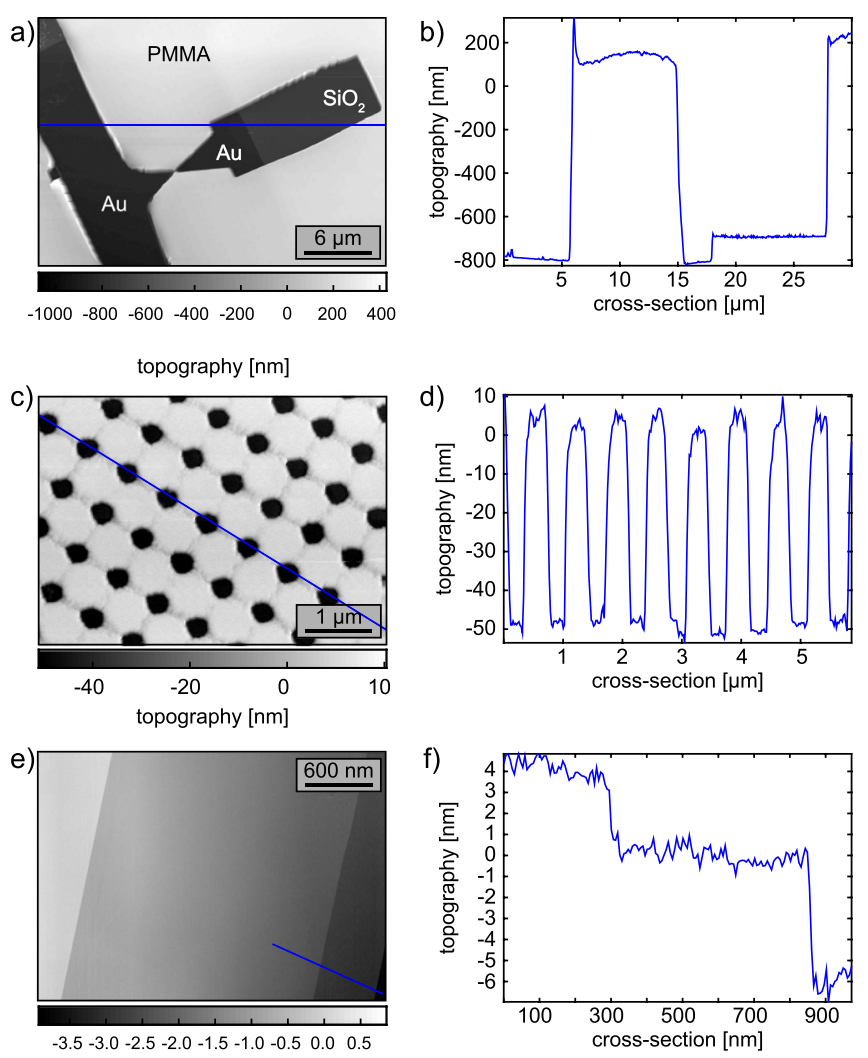
topography [nm]
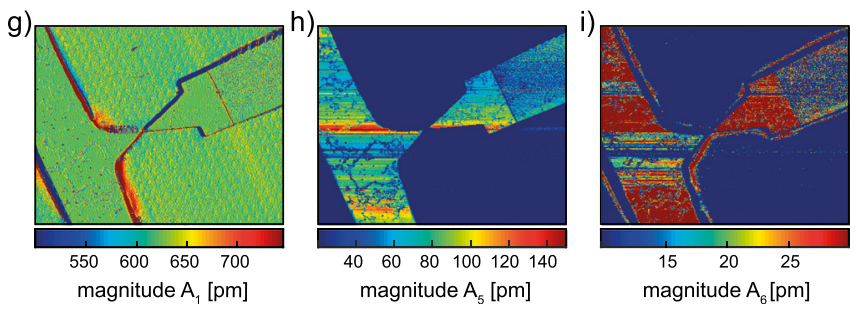

FIG. 2. (a) and (b) Topography and cross-section of a micro-fabricated sample with deep trenches. (c) and (d) Topography and cross-section of a silicon oxide calibration grid with a x-y periodicity of $679 \mathrm{~nm}$ and 59 height recorded under vacuum conditions $\left(\sim 10^{-6}\right.$ mbar). (e) and (f) Topography and cross-section of a highly oriented pyrolytic graphite (HOPG). (g)-(i) High harmonics magnitude data recorded simultaneously with the data displayed in (a).

walls. These data were recorded with a $48 \mathrm{~N} / \mathrm{m}$ cantilever, ${ }^{15}$ a z-modulation frequency of $3 \mathrm{kHz}$, an oscillation amplitude of $50 \mathrm{~nm}$, and a first harmonic magnitude setpoint of $620 \mathrm{pm}$. To demonstrate the vertical resolution of our off-resonance intermittent contact method, a highly oriented pyrolytic graphite (HOPG) surface was measured [Fig. 2(e)] with a $32 \mathrm{~N} / \mathrm{m}$ cantilever, ${ }^{15}$ a z-modulation frequency of $1.3 \mathrm{kHz}$, and an amplitude of $40 \mathrm{~nm}$. Single atomic steps can easily be detected [Fig. 2(f)]. The measurement results performed under vacuum are displayed in Figs. 2(c) and 2(d) using a $42 \mathrm{~N} / \mathrm{m}$ cantilever, ${ }^{15}$ a z-modulation frequency of $0.8 \mathrm{kHz}$, and an amplitude of $60 \mathrm{~nm}$.

The Zurich Instruments multi-channel LIA ${ }^{11}$ used here allows the detection of the magnitudes and phases of five higher harmonics in addition to those at the modulation frequency. While the fundamental mode magnitude $A_{1}$ [Fig. $2(\mathrm{~g})$, error signal] is kept constant at $620 \mathrm{pm}$ by the z-feedback to map the sample topography [Fig. 2(a)], the higher harmonic magnitudes [here $A_{5}$ and $A_{6}$ displayed in Figs. 2(h) and 2(i), 
respectively] are governed by the sharp features in the time trace signal [Fig. 1(c)] occurring during the contact of the tip with the surface of the sample. The higher harmonic magnitudes can thus be used to distinguish between the different materials at the surface of the sample. ${ }^{16}$ The PMMA and the $\mathrm{SiO}_{2}$ or $\mathrm{Au}$ appear clearly different in the 5thharmonic [Fig. 2(h)], while in the 6th-harmonic [Fig. 2(i)] a noticeable materials contrast occurs between the Au-coated and the bare $\mathrm{SiO}_{2}$-substrate. Note that in the peak force mode material, contrast images are obtained from fitting models for the tip-sample contact mechanics to force versus penetration data. For low peak forces required for imaging soft parts of the sample surface, only a small tip penetration into the sample occurs at locations with a high elastic modulus. Further, the penetration is obtained from the difference between the z-travel and the cantilever deflection. Small errors in either of the measured quantities thus lead to correspondingly large errors of the penetration data. The automated fitting of models of the contact mechanics to the data then becomes challenging and often fails completely. This is also the case for the operation mode presented here, but in contrast to the peak force mode, qualitative materials contrast images can still be obtained (c.f. simulation results displayed in Fig. S3 of the supplementary material).

Another example of materials contrast appearing in the higher harmonic magnitudes is displayed in Fig. 3. The topography of the polystyrene (PS)/low density polyethylene (LDPE) polymer blend sample [Fig. 3(a)] was recorded with a modulation frequency of $3 \mathrm{kHz}$ and an amplitude of $240 \mathrm{~nm}$ with a cantilever with a nominal stiffness of $40 \mathrm{~N} / \mathrm{m} .{ }^{15}$ Again,
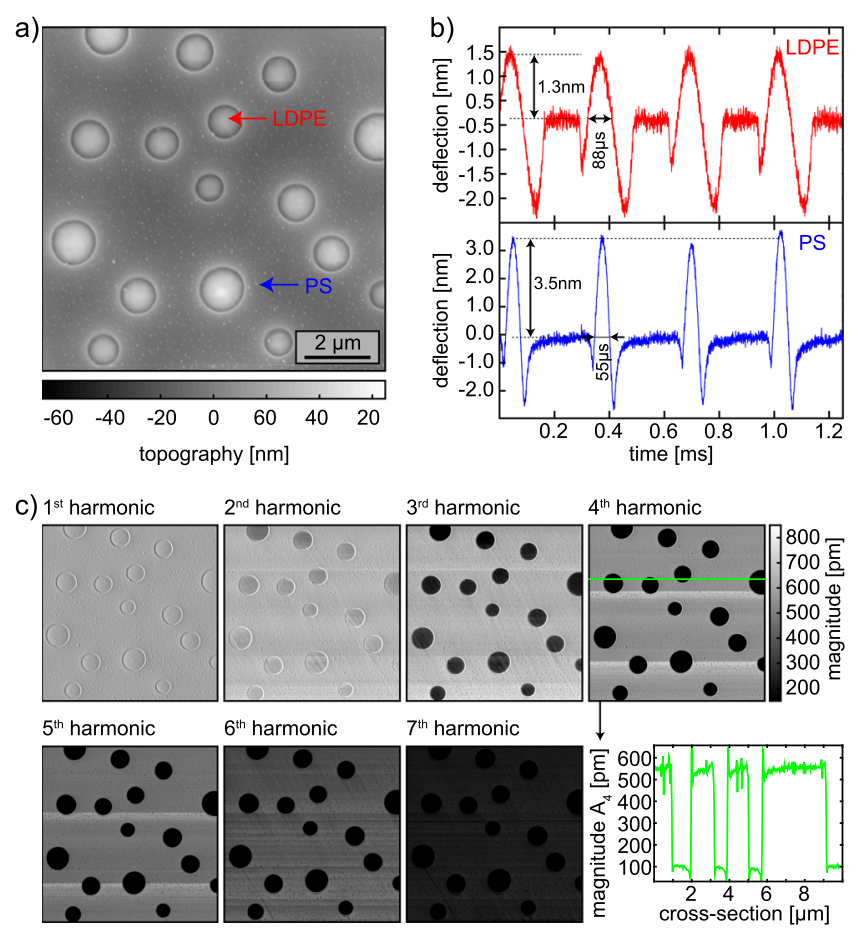

FIG. 3. (a) Topography image of a polymer blend sample consisting of polystyrene (PS) and low density polyethylene (LDPE). (b) Cantilever-deflection time trace signals recorded at the LDPE and PS positions indicated by the red and blue arrows in (a), respectively. (c) 1st-harmonic magnitude (error signal) and 6 higher harmonics. A cross-section of the 4th-harmonic displays distinct materials' contrast. the first harmonic magnitude was kept constant at $600 \mathrm{pm}$ by the z-feedback [Fig. 3(c), 1st harmonic].

Time traces of the cantilever deflection displayed in Fig. 3(b) were recorded at the positions marked in Fig. 3(a) by the blue (PS) and red (LDPE) arrows, respectively. The PS time trace [blue curve in Fig. 3(b)] shows a noticeably larger maximum cantilever deflection amplitude $A_{\text {peak }} \approx 3.5 \mathrm{~nm}$ than the $1.3 \mathrm{~nm}$ recorded on the LDPE [compare the vertical black arrows in Fig. 3(b)]. The fundamental mode magnitude $A_{1}$ nevertheless remains constant [Fig. 3(c)]. This is possible because the larger cantilever deflection on the PS occurs over a shorter time period $\left(\Delta t_{c}^{\mathrm{PS}} \approx 55 \mu \mathrm{s}<\Delta t_{c}^{\mathrm{LDPE}} \approx 88 \mu \mathrm{s}\right)$. The local contact force is thus not constant. This may appear as a disadvantage compared to the peak-force intermittent contact mode. However, in our operation mode, the highest contact force occurs at the location of the hardest material, c.f. $F_{\text {peak }}^{\mathrm{PS}} \approx 140 \mathrm{nN}>F_{\text {peak }}^{\mathrm{LDPE}} \approx 52 \mathrm{nN}$. This is true for all setpoints [see the simulation results displayed in Fig. S2 of the supplementary material].

Nevertheless, the contact forces observed here are high. They could however be reduced by lowering the first harmonic magnitude set-point or the $\mathrm{z}$ oscillation amplitude. Alternatively, a softer cantilever could be used, an option we have chosen for recording the data displayed in Fig. 4.

Note that the time traces of the deflection signal shown in Fig. 3(b) were recorded solely for illustration and discussion of the measurement physics, but are not required for the off-resonance intermittent contact operation mode presented here. All relevant information contained in the time traces is available if a sufficiently large number of higher harmonic magnitude and phases are recorded.

The time domain deflection signal can then be reconstructed by the Fourier series

$$
F(t)=\operatorname{Re}\left\{\sum_{k} A_{k} \cdot e^{i\left(2 \pi f_{k}+\theta_{k}\right)}\right\}
$$

where $f_{k}$ is the $k$ th harmonic frequency, and $A_{k}$ and $\phi_{k}$ are the $k$ th harmonic magnitude and phase. For the PS/LDPE polymer blend sample [Fig. 4(a)], eighteen magnitude and phase signals (supplementary material Fig. S1) were recorded together with the topography using three synchronized Zurich Instruments LIA. A cantilever with a stiffness of $0.4 \mathrm{~N} / \mathrm{m},{ }^{18}$ a modulation frequency of $1 \mathrm{kHz}$, a sample oscillation amplitude of $79 \mathrm{~nm}$, and a first harmonic magnitude setpoint of $1 \mathrm{~nm}$ were used. The cantilever deflection time traces are then reconstructed at all image points. Example data obtained at the positions in the PS and LDPE highlighted by the blue and red arrows in Fig. 4(a), respectively, are displayed in Fig. 4(b). Again, the peak deflection on the PS is larger than that on the LDPE [compare to results shown in Fig. 3(b)]. For comparison, the time traces measured with the digital oscilloscope [thin blue and red curves in Fig. 4(b)] are overlaid onto the reconstructed data. Excellent agreement is obtained except at positions where the cantilever deflection signal changes rapidly, e.g., when the tip snaps to the sample surface. Such a snap-to-contact occurs in less than $10 \mu \mathrm{s}$ and is thus much shorter than the period time of the 18th-harmonic which is $55 \mu \mathrm{s}$.

Using Eq. (1), the cantilever deflection signal, and from it the peak force can be calculated for each xy-point of the 

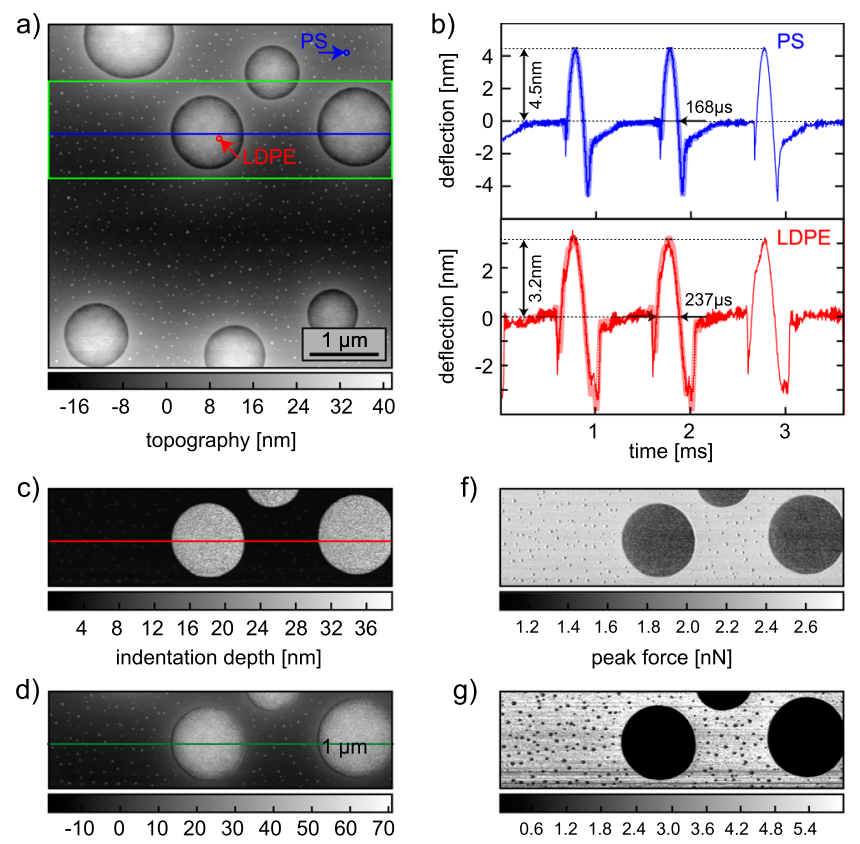

corrected topography [nm]

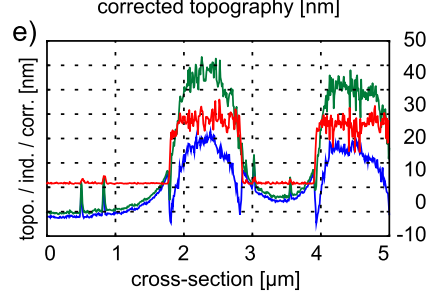

f)

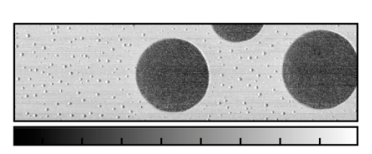

$\begin{array}{llllllll}1.2 & 1.4 & 1.6 & 1.8 & 2.0 & 2.2 & 2.4 & 2.6\end{array}$ peak force $[\mathrm{nN}]$

g)

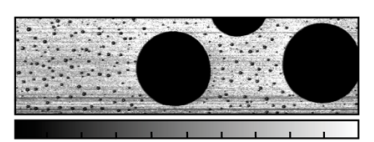

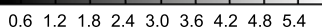

E-module [GPa]

h)

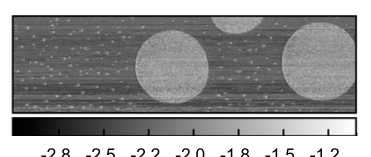

adhesion force $[\mathrm{nN}]$

FIG. 4. (a) As-recorded topography. (b) Typical cantilever-deflection time trace signals reconstructed from the 18 harmonic magnitude and phase values at the PS (red wide line) and LDPE (blue wide line) displayed together with time trace signals recorded directly with a digital oscilloscope (fine blue and red lines). (c) Spatial map of the indentation depth. (d) Corrected topography image. (e) Comparison of cross-sections of the as-measured topography (blue line), indentation depth (red line), and corrected topography (green line). The locations of the cross sections are indicated by the blue, red, and green lines in panels (a), (c), and (d), respectively. (f) Map of the peak force. (g) Image of the local Young's moduli. (h) Image of the local adhesion force.

image [Fig. 4(f)]. The indentation depth into the sample is then obtained from the difference of the cantilever deflection and the travel of sample z-position from the point of tipsample contact to the maximum oscillation amplitude. As expected, the indentation of the tip at the PS is only a few nanometers while it enters about $30 \mathrm{~nm}$ into the softer LDPE. The data shown in Fig. 4(a) thus only is a distorted measure of the sample topography. Improved topography data [Fig. $4(d)$ ] can be obtained from the latter by adding the local indentation depths displayed in Fig. 4(c). For comparison, cross-sections of the distorted topography, the indentation, and the corrected topography, and the corresponding crosssections are plotted in Fig. 4(e).

Because the force versus sample travel distance and indentation versus sample travel distance curves are known at each point, the local Young's modulus of the sample [Fig. $4(\mathrm{~g})]$ can be obtained by fitting the force versus indentation curves $F(\delta)$, with a suitable mathematical description for the tip-sample contact mechanics. Here, we use the DerjaguinMuller-Toporov (DMT) model with the Sneddon correction for the pyramidal tip ${ }^{13,17,18}$

$$
F(\delta)=\frac{1}{\sqrt{2}} \frac{E}{1-\nu^{2}} \tan (\alpha) \delta^{2},
$$

where $\delta$ is the penetration of the tip into the sample, $E$ is the Young's modulus of the sample, $\nu$ is the Poisson ratio, and $\alpha$ is the opening angle of the conical tip. The Young's modulus was derived from the unloading process that is of purely elastic nature. For the LDPE and the PS, Young's moduli of $0.05 \mathrm{GPa}$ and $4.9 \mathrm{GPa}$ were obtained, respectively, for a tip radius taken from the specification of the cantilever manufacturer. Our hardness values differ from those reported by Garcia et al. ${ }^{9}$ which were $0.168 \mathrm{GPa}$ and $2.1 \mathrm{GPa}$ for the LDPE and PS, respectively, obtained for a calibrated tip geometry. Materials contrast is also available in the adhesion force map displayed in Fig. 4(h).

In summary, the off-resonance intermittent contact mode presented here is a robust AFM operation mode suitable for imaging in air and vacuum, but would work also in liquids (not shown here). Materials contrast can be obtained directly from selected higher harmonic magnitudes [see, for example, Figs. 2(h) and 2(i) or Fig. 3(c)]. The results of simulations performed with a cantilever stiffness of $48 \mathrm{~N} / \mathrm{m}$, a sample oscillation amplitude of $50 \mathrm{~nm}$, and a first harmonic magnitude setpoint of $620 \mathrm{pm}$ reveal that for such conditions, the cantilever penetrates $2.7 \mathrm{~nm}$ and $3.1 \mathrm{~nm}$ into the $\mathrm{Au}$ and $\mathrm{SiO}_{2}$, respectively. The difference between the penetration depths on the two materials thus remains smaller than $0.5 \mathrm{~nm}$ (see supplementary material Fig. S3). With a soft cantilever and a low maximum contact force ideally suited for the softer polymer material of the sample, obtaining material contrast from fitting the repulsive parts of the force curves on the two harder materials is even more challenging and the obtained hardness values will not be reliable, such that only noisy materials contrast images would be obtained in the peak force mode. In our mode however, the simulation reveals an about $15 \%$ difference of 6th harmonic magnitude for hard cantilevers $(48 \mathrm{~N} / \mathrm{m})$, clearly demonstrating that low-noise materials contrast images can be obtained.

If a sufficient number of high harmonic magnitudes and phases is recorded, the force-distance curves at all imaged points can be reconstructed, and from those, local elastic properties of the sample can be obtained. Note that even a two-channel lock-in system would allow the detection of multiple high harmonics, if these are measured sequentially instead of simultaneously: one channel would be used for feedback (e.g., 1st harmonic magnitude) and the second one for materials contrast through a conveniently chosen higher harmonic. If one wants to calculate the mechanical properties of the material, a multi-channel lock-in is then needed. ${ }^{11,14}$

The off-resonance intermittent contact mode described here is particularly advantageous for higher tapping frequencies, provided the tip-sample distance can be actuated with a sufficiently high frequency. The latter is possible by a photothermal actuation of the cantilever. ${ }^{12}$ With our method, the tapping frequency is not limited by the bandwidth of the data acquisition system, but solely by that of the deflection detection system of the used scanning force microscope, which will be above $1 \mathrm{MHz}$ even in older systems. Hence, tapping frequencies of $1 \mathrm{MHz}$ could be used even in older systems, and pixel frequencies from $100 \mathrm{kHz}$ up to $1 \mathrm{MHz}$, depending 
on the setting of integration time of the lock-in are feasible. In newer systems, for example with a deflection detection bandwidth of $16 \mathrm{MHz}$, our method would then permit the measurement of 16 harmonics for a $1 \mathrm{MHz}$ tapping frequency, which allows a reasonable reconstruction of the force curve and materials contrast imaging, even without further data processing.

Because lock-in amplifiers capable of recording multiple higher harmonics (Zurich Instruments ${ }^{11}$ or Intermodulation Products ${ }^{14}$ ) are readily available, almost any scanning force microscope could be upgraded for the implementation of the operation mode described here.

See supplementary material to visualize the eighteen magnitude and phase signals simultaneous recorded used in Fig. 4, for a theoretical study showing that the peak force depends monotonously on the 1st harmonic magnitude signal and for a theoretical example of materials' contrast using high harmonics.

We thank Günther Hobi for his help in connecting the digital outputs of the Zurich Instruments lock-ins to the FPGA card inputs of our control system and Zurich Instruments for their input on the synchronization of the three lock-in amplifiers.

${ }^{1}$ B. Pittenger, N. Erina, and C. Su, "Quantitative mechanical mapping at the nanoscale with PeakForce QNM," in Application Note AN128 (Bruker, 2010).

${ }^{2}$ T. J. Young, M. A. Monclus, T. L. Burnett, W. R. Broughton, S. L. Ogin, and P. A. Smith, "The use of the PeakForce ${ }^{\mathrm{TM}}$ quantitative nanomechanical mapping AFM-based method for high-resolution Young's modulus measurement of polymers," Meas. Sci. Technol. 22, 125703 (2011).

${ }^{3}$ D. Platz, E. A. Tholén, D. Pesen, and D. B. Haviland, "Intermodulation atomic force microscopy," Appl. Phys. Lett. 92, 153106 (2008).
${ }^{4}$ D. Platz, E. A. Tholén, C. Hutter, A. C. von Bieren, and D. B. Haviland, "Phase imaging with intermodulation atomic force microscopy," Ultramicroscopy 110, 573 (2010).

${ }^{5}$ H.-J. Butt, B. Cappella, and M. Kappl, "Force measurements with the atomic force microscope: Technique, interpretation and applications," Surf. Sci. Rep. 59, 1 (2005).

${ }^{6}$ S. S. Borysov, D. Platz, A. S. de Wijn, D. Forchheimer, E. A. Tholén, A. V. Balatsky, and D. B. Haviland, "Reconstruction of tip-surface interactions with multimodal intermodulation atomic force microscopy," Phys. Rev. B 88, 115405 (2013).

${ }^{7}$ R. W. Stark, T. Drobek, and W. M. Heckl, "Tapping-mode atomic force microscopy and phase-imaging in higher eigenmodes," Appl. Phys. Lett. 74, 3296 (1999).

${ }^{8}$ A. Raman, S. Trigueros, A. Cartagena, A. P. Z. Stevenson, M. Susilo, E. Nauman, and S. Antoranz Contera, "Mapping nanomechanical properties of live cells using multi-harmonic atomic force microscopy," Nat. Nanotechnol. 6, 809 (2011).

${ }^{9}$ E. T. Herruzo, A. P. Perrino, and R. Garcia, "Fast nanomechanical spectroscopy of soft matter," Nat. Commun. 5, 3126 (2014).

${ }^{10}$ A. P. Nievergelt, B. W. Erickson, N. Hosseini, J. D. Adams, and G. E. Fantner, "Studying biological membranes with extended range high-speed atomic force microscopy," Sci. Rep. 5, 11987 (2015).

${ }^{11}$ See www.zhinst.com/products/hf2li for "HF2LI Lock-in Amplifier, Zurich Instruments."

${ }^{12}$ A. P. Nievergelt, J. D. Adams, P. D. Odermatt, and G. E. Fantner, "Highfrequency multimodal atomic force microscopy," Beilstein J. Nanotechnol. 5, 2459 (2014).

${ }^{13}$ J. W. Harding and I. N. Sneddon, "The elastic stresses produced by the indentation of the plane surface of a semi-infinite elastic solid by a rigid punch," Proc. Cambridge Philos. Soc. 41, 16 (1945).

${ }^{14}$ See www.intermodulation-products.com for "Intermodulation Products AB."

${ }^{15} \mathrm{PPP}$ NCH cantilever $\left(125 \times 28 \times 3.8 \mathrm{\mu m}^{3}\right)$, Nanosensors.

${ }^{16}$ O. Sahin, G. Yaralioglu, R. Grow, S. F. Zappe, A. Atalar, C. Quate, and O. Solgaard, "High-resolution imaging of elastic properties using harmonic cantilevers," Sens. Actuator A 114, 183 (2004).

${ }^{17}$ M. Gultekinoglu, Y. Jin Oh, P. Hinterdorfer, M. Duman, D. Çatçat, and K. Ulubayram, "Nanoscale characteristics of antibacterial cationic polymeric brushes and single bacterium interactions probed by force microscopy," RSC Adv. 6, 17092 (2016).

${ }^{18}$ Scanasyst-Air cantilever $\mathrm{k}=0.4 \mathrm{~N} / \mathrm{m}$, Bruker. 